\title{
Cross-comparison of diet quality indices for predicting chronic disease risk: findings from the Observation of Cardiovascular Risk Factors in Luxembourg (ORISCAV-LUX) study
}

\author{
Ala'a Alkerwi ${ }^{1 *}$, Cédric Vernier ${ }^{1}$, Georgina E. Crichton ${ }^{1,2}$, Nicolas Sauvageot ${ }^{1}$, Nitin Shivappa $^{3}$ and \\ James R. Hébert ${ }^{3,4}$ \\ ${ }^{1}$ Centre de Recherche Public de la Santé, Centre d'Etudes en Santé, 1 A rue Thomas Edison, Strassen L-1445, Luxembourg \\ ${ }^{2}$ Nutritional Physiology Research Centre, School of Health Sciences BJ2-36, University of South Australia, City East Campus, \\ Adelaide, SA, Australia \\ ${ }^{3}$ Cancer Prevention and Control Program, University of South Carolina, Columbia, SC 29208, USA \\ ${ }^{4}$ Department of Epidemiology and Biostatistics, Arnold School of Public Health, University of South Carolina, Columbia, \\ SC 29208, USA \\ (Submitted 11 June 2014 - Final revision received 11 August 2014-Accepted 1 October 2014 - First published online 5 December 2014)
}

\section{Abstract}

The scientific community has become increasingly interested in the overall quality of diets rather than in single food-based or single nutrientbased approaches to examine diet-disease relationships. Despite the plethora of indices used to measure diet quality, there still exist questions as to which of these can best predict health outcomes. The present study aimed to compare the ability of five diet quality indices, namely the Recommendation Compliance Index (RCI), Diet Quality Index-International (DQI-I), Dietary Approaches to Stop Hypertension (DASH), Mediterranean Diet Score (MDS), and Dietary Inflammatory Index (DII), to detect changes in chronic disease risk biomarkers. Nutritional data from 1352 participants, aged 18-69 years, of the Luxembourg nationwide cross-sectional ORISCAV-LUX (Observation of Cardiovascular Risk Factors in Luxembourg) study, 2007-8, were used to calculate adherence to the diet quality index. General linear modelling was performed to assess trends in biomarkers according to adherence to different dietary patterns, after adjustment for age, sex, education level, smoking status, physical activity and energy intake. Among the five selected diet quality indices, the MDS exhibited the best ability to detect changes in numerous risk markers and was significantly associated with lower levels of LDL-cholesterol, apo B, diastolic blood pressure, renal function indicators (creatinine and uric acid) and liver enzymes (serum $\gamma$-glutamyl-transpeptidase and glutamate-pyruvate transaminase). Compared with other dietary patterns, higher adherence to the Mediterranean diet is associated with a favourable cardiometabolic, hepatic and renal risk profile. Diets congruent with current universally accepted guidelines may be insufficient to prevent chronic diseases. Clinicians and public health decision makers should be aware of needs to improve the current dietary guidelines.

Key words: Diet quality indices: Cross-comparison: Chronic disease markers

The scientific community has become increasingly interested in the overall quality of diets rather than in single food-based or single nutrient-based approaches to examine diet-disease relationships ${ }^{(1)}$. Many different indices of healthy eating have been developed over the last three decades; most of these have been developed to reflect adherence to the national dietary guidelines and diversity of healthy choices within core food groups $^{(2)}$. These indices are used to examine diets for several attributes concurrently; therefore, they are able to provide a measure of overall diet quality, which is not possible when only single nutrients or foods are examined ${ }^{(3)}$. Although every model of healthy eating has its own advantages and limitations, the general purpose of an index is to synthesise a large amount of dietary information as a single indicator useful for assessing putative risk factor-disease relationships ${ }^{(4)}$.

Numerous indices have been developed based on the American Dietary Guidelines ${ }^{(5-9)}$, while others have been developed with some adaptation to reflect the dietary guidelines of other countries in Europe ${ }^{(10-12)}$, Canada $^{(13)}$ and Australia ${ }^{(14)}$. Some indices have been developed around specific dietary

Abbreviations: BP, blood pressure; DASH, Dietary Approaches to Stop Hypertension; DBP, diastolic blood pressure; DII, Dietary Inflammatory Index; DQI-I, Diet Quality Index-International; GOT, glutamate oxaloacetate transaminase; GPT, glutamate-pyruvate transaminase; $\gamma$-GT, $\gamma$-glutamyl-transpeptidase; HbA1c, glycated Hb; HOMA-IR, homeostasis model assessment of insulin resistance; MDS, Mediterranean Diet Score; ORISCAV-LUX, Observation of Cardiovascular Risk Factors in Luxembourg; RCI, Recommendation Compliance Index.

*Corresponding author: Dr A. Alkerwi, fax +352 26970 719, email alaa.alkerwi@crp-sante.lu 
patterns, such as the Mediterranean diet ${ }^{(15)}$ and the Dietary Approaches to Stop Hypertension (DASH) diet ${ }^{(16)}$, while an international diet quality index has been developed for a cross-national comparison of diet quality ${ }^{(17)}$ and an index has been developed based on the available literature on various dietary components and inflammatory indices ${ }^{(18)}$.

Despite the plethora of indices used to measure diet quality, there still exist questions as to which of these can best predict health outcomes. Several systematic reviews on the differences in existing diet quality indices and their limitations have been published previously ${ }^{(2,3,19-21)}$, suggesting the need to assess the effectiveness of these tools in predicting health status. The construct validity of an index depends on the extent to which it is able to distinguish between individuals on certain relevant health-related intermediate markers, its association with health outcomes, and its capacity to predict the risk of chronic diseases or mortality ${ }^{(22)}$. Although several attempts have been made to validate individual dietary indicators ${ }^{(23,24)}$, very few studies have compared existing diet quality indices with regard to the biomarkers of diet and disease ${ }^{(25,26)}$ and most of these have focused on the Mediterranean Diet Score (MDS) ${ }^{(27,28)}$.

The objective of the present study was to compare the ability of five different diet quality indices, namely the Recommendation Compliance Index (RCI), Diet Quality Index-International (DQI-I), DASH score, MDS and Dietary Inflammatory Index (DII), to detect changes in chronic disease risk biomarkers. These diet quality indices were selected according to the fundamental diversity of how they were conceived and built, including their evidence-based scoring algorithms, attributes and adaptability to widespread (if not universal) application. They were developed by different groups of researchers and in diverse settings (Northern European, North American and Mediterranean populations). A deeper understanding of how well these diet quality indices relate to a set of risk markers could improve the success of public health messages and fulfil the demand of many nutrition researchers for a suitable measure of diet quality. As this is exploratory research, we did not advance a hypothesis of any index preference.

\section{Materials and methods}

\section{Study population}

The study population consisted of 1352 participants of the ORISCAV-LUX (Observation of Cardiovascular Risk Factors in Luxembourg) study, a nationwide cross-sectional populationbased survey of cardiovascular risk factors in Luxembourg. The sample size was based on a theoretically expected participation rate of $32.2 \%{ }^{(29)}$. The data collection procedures, sample design and representativeness of the ORISCAV-LUX study have been described in detail elsewhere ${ }^{(29,30)}$. In short, the stratified random sample was identified from the national insurance registry of non-institutionalised individuals aged 18-69 years. The participants were recruited between November 2007 and January 2009 following an invitation letter and phone contact inviting prospective participants to visit the study centre. Trained research staff provided the participants with detailed instructions on how to complete the self-administered questionnaire, assisted them in completing the dietary information, and then checked the correctness of responses.

Dietary intake was assessed by means of a validated 134item semi-quantitative $\mathrm{FFQ}^{(31,32)}$. Food intakes were calculated by multiplying the self-reported food portion by the frequency of consumption. Energy and nutrient intake data were compiled using the French Composition Table ${ }^{(33)}$. Information regarding demographic, socio-economic and lifestyle characteristics was collected as well as direct blood pressure (BP) and anthropometric measurements were taken. For the present study, data from 1352 participants were available after eliminating those with missing data on dietary habits.

The ORISCAV-LUX study was conducted according to the guidelines laid down in the Declaration of Helsinki. All procedures involving human subjects were approved by the National Research Ethics Committee and the National Commission for Private Data Protection. Written informed consent was obtained from all participants.

\section{Diet quality indices}

FFQ-based dietary intakes were used to calculate adherence to the five diet quality indices. The calculation of these indices is summarised in online Appendix 1a-e; however, detailed information can be found in the respective publications pertaining to each index. Briefly, the RCI ${ }^{(10)}$ has been developed previously to measure the degree of adherence to Luxembourg national dietary recommendations, which are consistent with the key prevailing European and international dietary guidelines. It is a food- and nutrient-based composite of thirteen components and ranges between -0.5 (due to a negative half point for excessive salt intake) and 14 ( 2 points for more daily fruit and vegetable servings) points, where a higher degree of adherence results in higher scores.

To include a more comprehensive tool describing and comparing the diversity of consumption between countries, the DQI-I suggested by Kim et al. ${ }^{(17)}$, which is based on the North American dietary guidelines, was selected. This index focuses on four major components: variety; adequacy; moderation; overall balance. Similarly, it incorporates both nutrients and foods from the assessed diet. The total DQI-I score ranges from 0 to 100 , where a score of ' 0 ' reflects a poorer-quality diet and a score of '100' reflects a high-quality diet. In the present study, meat and poultry were considered as one group to calculate the within-group variety component, as it was not possible to distinguish the intake according to the FFQ.

As a clinical profile-targeted index, the DASH score was calculated as originally designed by Fung et al. ${ }^{(16)}$ for BP reduction. Based on both foods and nutrients, the DASH score focuses on eight beneficial and detrimental items of the diet. For each of the items, the participants were classified into quintiles according to their intake ranking. Component scores for beneficial items (vegetables, fruits and nuts, legumes, low-fat dairy products and whole grains) were assigned a value of 1 for quintile 1 and a value of 5 for quintile 5. Reverse scores were assigned for detrimental items ( $\mathrm{Na}$, red and processed meats, and sweetened beverages). 
The component scores were summed to obtain an overall DASH score for each participant ranging from 8 to 40 points.

In contrast to other indices, a region-specific diet quality index, the modified version of the MDS, as described by Trichopoulou et al. ${ }^{(15)}$, was chosen to measure the degree of adherence to the traditional Mediterranean diet. It is characterised by nine food and nutrient components: high consumption of vegetables, fruits and nuts, pulses, cereals, and fish; high ratio of monounsaturated fat:saturated fat; low consumption of meat and dairy products; moderate consumption of alcohol. For beneficial components (vegetables, legumes, fruits and nuts, cereal and fish), consumption above the median was assigned a value of 1 ; otherwise a value of 0 was assigned. For components presumed to be detrimental (meat, poultry and dairy products), consumption equal to or above the median was assigned a value of 0 ; otherwise a value of 1 was assigned. For alcohol consumption, a value of 1 was assigned if the intake was between 10 and $50 \mathrm{~g} / \mathrm{d}$ for men and a value of 1 was assigned if the intake was between 5 and $25 \mathrm{~g} / \mathrm{d}$ for women. The total MDS ranged from 0 (minimal adherence to the traditional Mediterranean diet) to 9 (maximal adherence).

As a novel non-recommendation-based index selected for comparison, the DII focuses on the inflammatory properties of the diet. It is a literature-based index, involving the review of nearly 2000 articles published through 2010 on the effect of forty-five food parameters on six inflammatory biomarkers (IL-1 $\beta$, IL-4, IL-6, IL-10, TNF- $\alpha$ and C-reactive protein). It is scored according to whether the dietary parameter increased $(+1)$, decreased $(-1)$ or had no $(0)$ effect on each of the six inflammatory biomarkers ${ }^{(18)}$. In contrast to the other diet quality indices, higher values of the DII indicate a pro-inflammatory (i.e. less healthy) dietary profile, whereas lower values indicate an anti-inflammatory (i.e. more healthy) dietary profile.

\section{Assessment of risk markers}

Several anthropometric, clinical and plasma markers were examined, including glycaemic biomarkers (plasma glucose, serum insulin and glycated $\mathrm{Hb}(\mathrm{HbA1c})$ and homeostasis model assessment of insulin resistance (HOMA-IR)); lipid biomarkers (total cholesterol, LDL-cholesterol, HDL-cholesterol, TAG, apo A1 and apo B); cardiometabolic markers (BMI, waist circumference, systolic BP, diastolic blood pressure (DBP) and high-sensitivity serum C-reactive protein); hepatic function biomarkers (serum $\boldsymbol{\gamma}$-glutamyl-transpeptidase $(\boldsymbol{\gamma}$-GT), serum glutamate oxaloacetate transaminase (GOT) and serum glutamate-pyruvate transaminase (GPT)); renal function biomarkers (creatinine and uric acid); and anaemic biomarkers ( $\mathrm{Hb}$ and haematocrit).

BMI was calculated as weight in $\mathrm{kg}$ divided by height in $\mathrm{m}^{2}$ $\left(\mathrm{kg} / \mathrm{m}^{2}\right)$. BP was measured using an Omron ${ }^{\circledR}$ MX3 Plus automated oscillometric blood pressure monitor (O-HEM-742-E). Measurements were taken at least three times with the participants in a seated position and with a minimum interval of 5 min between each measurement. The average of the last two readings was used in the analysis. HOMA-IR was calculated as follows:

HOMA-IR $=($ fasting glucose $\times$ fasting insulin $/ 22 \cdot 5)$.
All the biomarkers were measured according to ORISCAVLUX standardised protocols and have been described in detail in previous publications ${ }^{(10,30,34)}$.

\section{Assessment of other variables}

Self-reported time spent engaging in both moderate and intense physical activity was obtained from the International Physical Activity Questionnaire ${ }^{(35)}$, from which physical activity in metabolic equivalents-h/week was calculated. Detailed information regarding smoking status was obtained from the health questionnaire, and each participant was classified as a smoker or a non-smoker.

\section{Statistical analyses}

For descriptive purposes, the five diet quality indices were divided into quartiles (Q). The Q1 and Q4 of each index are reported to compare the demographic, socio-economic and lifestyle characteristics of the participants. The number and percentage of participants are reported for categorical variables and means and standard errors are reported for continuous symmetrically distributed variables; otherwise medians and interquartile ranges are reported. The $P$ values were computed from the $\chi^{2}$ test, one-way ANOVA and Kruskal-Wallis test.

The bivariate Spearman correlation method was used to assess the inter-correlation between the diet quality indices and their correlations with several micronutrients and vitamins, as this method is not influenced by the outlying values and results are therefore more robust in meeting normality assumptions.

As the diet quality indices have disparate scoring systems, raw scores of each diet quality index were standardised into $z$-scores, to enable meaningful cross-index comparisons.

To assess the independent linear associations between each diet quality index and risk biomarkers, multiple linear regression analyses were conducted to calculate $\beta$-regression coefficients for each $1-z$ score difference across the diet quality indices. Each diet quality index (explanatory variable) was modelled to examine the independent associations with different biomarkers (outcome), after adjustment for a number of potential covariates: age; sex; education level (primary, secondary or tertiary), smoking status (current, former or non-smoker); physical activity (metabolic equivalents-h/week); total energy intake $(\mathrm{kcal} / \mathrm{d})$. As total energy intake is included in the construction of the DII, this variable was not included in the respective analyses. These covariates were identified as major known determinants of health based on the literature. As several of the outcomes were not normally distributed, including BMI, systolic BP, C-reactive protein, TAG, $\gamma$-GT, GOT, GPT, all glycaemic biomarkers, and lipid biomarkers, except total cholesterol, generalised linear models with a $\gamma$-distribution and logarithm as the link function were used. Otherwise ANCOVA models were fit with special attention being paid to the homogeneity of variance and covariance assumptions.

Of the initial sample of 1352 participants, those who reported dieting for weight loss were excluded ( $n$ 199) from all the multivariable models. Participants taking antidiabetic ( $n$ 43), hypotensive ( $n$ 187) and lipid-lowering ( $n$ 128) 
Table 1. Characteristics of the participants by quartiles† (Q) of five different diet quality indices, ORISCAV-LUX (Observation of Cardiovascular Risk Factors in Luxembourg) survey, 2007-8

(Number and percentage of participants; mean values and standard deviations; median values and interquartile ranges (IQR))

\begin{tabular}{|c|c|c|c|c|c|c|c|c|c|c|c|c|c|c|c|c|c|c|c|c|}
\hline \multirow[b]{3}{*}{ Participant characteristics } & \multicolumn{4}{|c|}{$\mathrm{RCl}(n$ 1234) } & \multicolumn{4}{|c|}{ DQI-I $(n$ 1206) } & \multicolumn{4}{|c|}{ DASH score $(n 1285)$} & \multicolumn{4}{|c|}{ MDS ( $n$ 1352) } & \multicolumn{4}{|c|}{ DII ( $n$ 1352) } \\
\hline & \multicolumn{2}{|c|}{ Q1 (n‡ 302) } & \multicolumn{2}{|c|}{ Q4 $(n \ddagger 287)$} & \multicolumn{2}{|c|}{ Q1 (n‡ 301) } & \multicolumn{2}{|c|}{ Q4 $(n \ddagger 301)$} & \multicolumn{2}{|c|}{ Q1 (n‡ 321) } & \multicolumn{2}{|c|}{ Q4 (n‡ 321) } & \multicolumn{2}{|c|}{ Q1 $(n \ddagger 401)$} & \multicolumn{2}{|c|}{ Q4 (n‡ 313) } & Q1 ( & 338) & Q4 $(r$ & 338) \\
\hline & $n$ & $\%$ & $n$ & $\%$ & $n$ & $\%$ & $n$ & $\%$ & $n$ & $\%$ & $n$ & $\%$ & $n$ & $\%$ & $n$ & $\%$ & $n$ & $\%$ & $n$ & $\%$ \\
\hline Age (years) & & & & & & & & & & & & & & & & & & & & \\
\hline Mean & & & & & & & 48. & & & & 46. & & & & 46. & & & & & \\
\hline SD & & & & & & & 0 & & & & 0 & & & & 0 & & & & & \\
\hline Sex & & & & & & & & & & & & & & & & & & & & \\
\hline Men & 148 & 49.0 & 129 & 44.9 & 179 & 59.5 & $116^{\star \star \star}$ & 38.5 & 185 & 57.6 & $121^{\star \star \star}$ & 37.7 & 195 & 48.6 & 137 & 43.8 & 172 & 50.9 & 153 & $45 \cdot 3$ \\
\hline Women & 154 & 51.0 & 158 & $55 \cdot 1$ & 122 & 40.5 & 185 & 61.5 & 136 & 42.4 & 200 & $62 \cdot 3$ & 206 & $51 \cdot 1$ & 176 & $56 \cdot 2$ & 166 & 49.1 & 185 & 54.7 \\
\hline Education level & & & & & & & & & & & & & & & & & & & & \\
\hline Primary & 83 & $27 \cdot 7$ & 71 & 24.9 & 74 & 24.6 & 73 & $24 \cdot 3$ & 84 & $26 \cdot 3$ & 90 & $28 \cdot 2$ & 90 & $22 \cdot 7$ & $93^{*}$ & 29.8 & 80 & 23.9 & 88 & 26.6 \\
\hline Secondary & 145 & $48 \cdot 3$ & 127 & 44.6 & 150 & 49.8 & 139 & 46.6 & 161 & 50.3 & 136 & 42.6 & 196 & 49.5 & 142 & 45.5 & 164 & 49.0 & 157 & 47.4 \\
\hline Tertiary & 72 & 24.0 & 87 & 30.5 & 77 & $25 \cdot 6$ & 86 & 28.9 & 75 & 23.4 & 93 & 29.2 & 110 & 27.8 & 77 & 24.7 & 91 & $27 \cdot 1$ & 86 & 26.0 \\
\hline Smoking status & & & & & & & & & & & & & & & & & & & & \\
\hline Non-smoker & 218 & $72 \cdot 2$ & $257^{\star \star \star}$ & 89.5 & 205 & 68.1 & $260^{\star \star \star}$ & 86.4 & 226 & 70.4 & $276^{\star \star \star}$ & 86.0 & 304 & 75.8 & 249 & 79.6 & 273 & 80.8 & 257 & 76.0 \\
\hline Smoker & 84 & 27.8 & 30 & 10.5 & 96 & 31.9 & 41 & 13.6 & 95 & 29.6 & 45 & 14.0 & 97 & 24.2 & 64 & 20.4 & 65 & $19 \cdot 2$ & 81 & $24 \cdot 0$ \\
\hline Alcohol intake & & & & & & & & & & & & & & & & & & & & \\
\hline No & 37 & $12 \cdot 3$ & 46 & $16 \cdot 0$ & 30 & $10 \cdot 0$ & 60 & 19.9 & 35 & 10.9 & $64^{* *}$ & 19.9 & 53 & $13 \cdot 2$ & 47 & $15 \cdot 0$ & 60 & 17.8 & 38 & $11 \cdot 2$ \\
\hline$>0-3$ drinks/d & 207 & 68.5 & 188 & 65.5 & 203 & 67.4 & 176 & 58.5 & 211 & 65.7 & 195 & 60.7 & 276 & 68.8 & 201 & $64 \cdot 2$ & 196 & 58.0 & 243 & 71.9 \\
\hline$>3$ drinks $/ \mathrm{d}$ & 58 & $19 \cdot 2$ & 53 & 18.5 & 68 & 22.6 & 65 & 21.6 & 75 & $23 \cdot 4$ & 62 & $21 \cdot 1$ & 72 & 18.0 & 65 & 20.8 & 82 & $24 \cdot 2$ & 57 & 16.9 \\
\hline Importance of balanced $m$ & & & & & & & & & & & & & & & & & & & & \\
\hline Very important & 118 & 39.1 & $183^{\star \star \star}$ & 63.8 & 111 & 36.9 & $190^{\star \star \star}$ & 63.1 & 124 & 38.6 & $219^{\star \star \star}$ & 68.2 & 179 & 44.7 & $192^{\star \star \star}$ & 61.3 & 192 & $57 \cdot 0$ & $149^{\star \star}$ & $44 \cdot 1$ \\
\hline Enough & 154 & 51.0 & 100 & 34.8 & 147 & 48.8 & 104 & 34.6 & 153 & 47.7 & 97 & 30.2 & 181 & $45 \cdot 1$ & 113 & $36 \cdot 1$ & 128 & 38.0 & 162 & 47.9 \\
\hline Little & 30 & $9 \cdot 9$ & 4 & 1.4 & 43 & $14 \cdot 3$ & 7 & $2 \cdot 3$ & 44 & 13.7 & 5 & $1 \cdot 6$ & 41 & $10 \cdot 2$ & 8 & $2 \cdot 6$ & 17 & $5 \cdot 0$ & 26 & 8.0 \\
\hline Under dieting & & & & & & & & & & & & & & & & & & & & \\
\hline Yes & 29 & $9 \cdot 6$ & $60^{\star \star \star}$ & $21 \cdot 1$ & 32 & $10 \cdot 6$ & $56^{*}$ & $18 \cdot 7$ & 30 & $9 \cdot 4$ & $64^{\star \star \star}$ & 20.0 & 50 & 12.5 & $63^{\star \star}$ & $20 \cdot 2$ & 62 & 18.5 & $31^{\star \star \star}$ & $9 \cdot 2$ \\
\hline $\begin{array}{l}\text { lypertension treatment } \\
\text { Yes }\end{array}$ & 35 & 11.6 & $48^{*}$ & $16 \cdot 7$ & 28 & 9.3 & $51^{\star \star \star}$ & $16 \cdot 9$ & 30 & $9 \cdot 3$ & $48^{*}$ & $15 \cdot 0$ & 43 & 10.7 & $53^{*}$ & 16.9 & 53 & $15 \cdot 7$ & 47 & 13.9 \\
\hline Dyslipidaemia treatment & & & & & & & & & & & & & & & & & & & & \\
\hline Yes & 19 & $6 \cdot 3$ & $37^{\star \star}$ & $12 \cdot 9$ & 16 & 5.3 & $46^{\star \star \star}$ & $15 \cdot 3$ & 25 & 7.8 & $43^{*}$ & 13.4 & 28 & 7.0 & $39^{* *}$ & 12.5 & 36 & 10.7 & 27 & 8.0 \\
\hline Diabetes treatment & & & & & & & & & & & & & & & & & & & & \\
\hline Yes & 7 & $2 \cdot 3$ & 13 & 4.5 & 9 & 3.0 & 14 & 4.7 & 7 & $2 \cdot 2$ & 14 & 4.4 & 8 & 2.0 & $13^{*}$ & $4 \cdot 2$ & 14 & $4 \cdot 1$ & 10 & 3.0 \\
\hline Physical activity (MET-h/w & & & & & & & & & & & & & & & & & & & & \\
\hline Median & & & & & & & 28 & & & & 36 & & & & 405 & & & & 283 & \\
\hline IQR & & & & & & & 40 & & & & 47 & & & & 45 & & & & 4 & \\
\hline Energy & & & & & & & & & & & & & & & & & & & & \\
\hline $\mathrm{kJ} / \mathrm{d}$ & & & & & & & & & & & & & & & & & & & & \\
\hline Median & & & 872 & & & & 853 & & & & 866 & & & & 975 & & & & & \\
\hline IQR & & & & & & & 34 & & & & 42 & & & & 44 & & & & & \\
\hline $\mathrm{kcal} / \mathrm{d}$ & & & & & & & & & & & & & & & & & & & & \\
\hline Median & & & & & & & 203 & & & & 207 & & & & 233 & & & & 16 & \\
\hline IQR & & & & & & & 83 & & & & 10 & & & & 10 & & & & & \\
\hline Protein (\%E) & & & & & & & & & & & & & & & & & & & & \\
\hline Median & & & & & & & 15 & & & & 15 & & & & 15 & & & & & \\
\hline IQR & & & & & & & 2 & & & & 2 & & & & 2 & & & & & \\
\hline Fats (\%E) & & & & & & & & & & & & & & & & & & & & \\
\hline Median & & & & & & & 33 & & & & 35 & & & & 37 & & & & & \\
\hline IQR & & & & & & & 1 & & & & $\varepsilon$ & & & & s & & & & & \\
\hline Carbohydrates (\%E) & & & & & & & & & & & & & & & & & & & & \\
\hline Median & & & 46 & & & & 46. & & & & 45 & & & & 43 & & & & 42 & \\
\hline IQR & & & & & & & 1 & & & & 1 & & & & s & & & & & \\
\hline
\end{tabular}

RCI, Recommendation Compliance Index; DQI-I, Diet Quality Index-International; DASH, Dietary Approaches to Stop Hypertension; MDS, Mediterranean Diet Score; DII, Dietary Inflammatory Index; MET, metabolic equivalents; $\%$, percentage of total daily energy intake.

Values were significantly different from that for Q1: ${ }^{*} P<0.05,{ }^{* *} P<0.01,{ }^{* \star *} P<0.001$ ( $\chi^{2}$ test for categorical variables and one-way ANOVA for continuous normally distributed variables; otherwise from the Kruskal-Wallis test).

tOnly Q1 and Q4 of each diet quality index are given in the table.

$\ddagger$ Difference in the number of cases is related to missing values for several variables. 
medications were excluded from the following models, respectively: glucose; systolic $\mathrm{BP}$ and DBP; lipid biomarkers. Non-fasting participants ( $n$ 26) were excluded from the glycaemic and lipid biomarker models. Hence, the final sample size used in the multivariable analyses varied between 1007 and 1153 participants.

Several sensitivity analyses were conducted to establish the robustness of the study results. First, the interaction terms for age $\times$ diet quality scores and sex $\times$ diet quality scores were tested. Analyses stratified by sex (male and female) and age group ( $<50$ and $\geq 50$ years) were conducted (results summarised below). Second, controlling for self-reported health awareness on the importance of eating a balanced diet did not change the findings. Similarly, results were similar when participants taking medications and dieting for weight loss were included in the analyses.

The results were considered significant at the $5 \%$ critical level ( $P<0 \cdot 05$, two-sided). All statistical analyses were conducted using PASW $^{(}$for Windows $^{(\mathcal{O}}$ version 21.0 software (formerly SPSS Statistics, Inc.).

\section{Results}

\section{Characteristics of participants according to} diet quality indices

Table 1 summarises the characteristics of participants according to the quartile of each diet quality index, where Q1 reflects the lowest adherence and Q4 reflects the highest adherence to each specific dietary pattern, except for the DII, for which a reverse pattern exists (i.e. it ranges from a more anti-inflammatory (Q1) profile to a more pro-inflammatory profile (Q4)). Participants with better diet quality scores on all the indices were significantly older. A significant sex difference was observed for the DQI-I and DASH patterns, with women having higher scores than men. Globally, the five diet quality indices varied remarkably with regard to lifestyle behaviours. Participants with better diet quality scores were less likely to smoke and engaged in more physical activity, with the exception of the DQI-I. Across the five diet quality indices, participants exhibiting higher adherence tended to be more aware of the importance of balanced meals.

Participants with higher diet quality scores reported lower total energy intakes, except for the Mediterranean dietary pattern. With increasing scores, the percentage of total energy intake per $d$ derived from fat decreased, but that derived from carbohydrates increased (with the exception of the DII). Energy intake derived from protein did not differ considerably according to dietary pattern.

\section{Correlations between diet quality indices and micronutrients}

The correlations of the five diet quality indices with one another and with micronutrients are given in Table 2. The five diet quality indices were significantly correlated with one another ( $\rho$ values ranged between $|0 \cdot 28|$ and $|0 \cdot 65|$ and all $P<0 \cdot 0001)$. As expected, negative correlations were observed between the DII and the other four indices due to the reverse DII scoring. All the indices were strongly correlated with the intakes of the three types of fatty acids and to the intakes of

Table 2. Spearman's correlation coefficients $(r)$ among the diet quality indices and essential macronutrients and micronutrients, ORISCAV-LUX (Observation of Cardiovascular Risk Factors in Luxembourg) study, 2007-8

\begin{tabular}{|c|c|c|c|c|c|}
\hline & $\mathrm{RCl}(n+1197-1234)$ & DQI-I (n† 1196-1206) & DASH score (n† 1269-1285) & MDS (n† 1331-1352) & DII (n† 1331-1344) \\
\hline DQI-I & $0.56^{\star \star \star}$ & - & - & - & - \\
\hline DASH score & $0.55^{\star \star \star}$ & $0.65^{\star \star \star}$ & - & - & - \\
\hline MDS & $0.35^{\star \star \star}$ & $0.31^{\star \star \star}$ & $0.32^{\star \star \star}$ & - & - \\
\hline DII & $-0 \cdot 28^{\star \star \star}$ & $-0.40^{\star \star \star}$ & $-0 \cdot 34^{\star \star \star}$ & $-0.46^{\star \star \star}$ & - \\
\hline SFA & $-0.45^{\star \star \star}$ & $-0 \cdot 29^{\star \star \star}$ & $-0 \cdot 30^{\star \star \star}$ & $-0.06^{\star}$ & $-0.40^{\star \star \star}$ \\
\hline MUFA & $-0.44^{\star \star \star}$ & $-0 \cdot 27^{\star \star \star}$ & $-0 \cdot 27^{\star \star \star}$ & $0 \cdot 11^{\star \star \star}$ & $-0.47^{\star \star \star}$ \\
\hline PUFA & $-0 \cdot 29^{\star \star \star}$ & $-0 \cdot 11^{\star \star \star}$ & $-0 \cdot 15^{\star \star \star}$ & $0 \cdot 17^{\star \star \star}$ & $-0.60^{\star \star \star}$ \\
\hline Vitamin A & $-0 \cdot 23^{\star \star \star}$ & $-0 \cdot 19^{\star \star \star}$ & $-0 \cdot 16^{\star \star \star}$ & 0.01 & $-0.43^{\star \star \star}$ \\
\hline Vitamin $\mathrm{B}_{1}$ & 0.03 & $0 \cdot 15^{\star * *}$ & $0 \cdot 13^{\star \star \star}$ & $0 \cdot 20^{* \star *}$ & $-0.74^{\star \star \star}$ \\
\hline Vitamin $B_{2}$ & -0.05 & $0.08^{\star *}$ & $0 \cdot 14^{\star \star \star}$ & $0.06^{*}$ & $-0.68^{\star \star \star}$ \\
\hline Vitamin $B_{3}$ & -0.03 & 0.02 & -0.04 & $0 \cdot 13^{\star \star \star}$ & $-0.64^{\star \star \star}$ \\
\hline Vitamin $B_{5}$ & 0.00 & $0 \cdot 11^{\star * *}$ & $0 \cdot 13^{\star \star \star}$ & $0 \cdot 15^{\star \star \star}$ & $-0.74^{\star \star \star}$ \\
\hline Vitamin $B_{6}$ & $0.09^{\star \star \star}$ & $0 \cdot 20^{\star * *}$ & $0 \cdot 17^{\star \star \star}$ & $0.25^{\star \star \star}$ & $-0.79^{\star \star \star}$ \\
\hline Vitamin $\mathrm{B}_{9}$ & $0 \cdot 24^{\star \star \star}$ & $0.36^{\star \star \star}$ & $0.33^{\star \star \star}$ & $0.38^{\star \star \star}$ & $-0.88^{\star \star}$ \\
\hline Vitamin $\mathrm{B}_{12}$ & $-0.09^{\star \star \star}$ & $-0 \cdot 15^{\star \star \star}$ & $-0 \cdot 15^{\star \star \star}$ & $0 \cdot 15^{\star \star \star}$ & $-0.47^{\star \star \star}$ \\
\hline Vitamin C & $0 \cdot 28^{\star \star \star}$ & $0.49^{\star * \star}$ & $0.44^{\star \star \star}$ & $0.37^{\star \star \star}$ & $-0.69^{\star \star \star}$ \\
\hline Vitamin D & 0.03 & -0.03 & -0.03 & $0.23^{\star \star \star}$ & $-0.45^{\star \star \star}$ \\
\hline Vitamin E & $-0 \cdot 21^{\star \star *}$ & -0.05 & $-0 \cdot 10^{\star \star \star}$ & $0 \cdot 19^{* * *}$ & $-0.61^{\star \star \star}$ \\
\hline $\mathrm{Ca}$ & 0.03 & $0.13^{\star \star \star}$ & $0 \cdot 23^{\star \star *}$ & 0.03 & $-0.56^{\star \star \star}$ \\
\hline $\mathrm{Fe}$ & -0.05 & 0.05 & 0.02 & $0 \cdot 21^{\star \star \star}$ & $-0.72^{\star \star \star}$ \\
\hline I & -0.02 & 0.00 & 0.05 & $0.12^{\star \star \star}$ & $-0.52^{\star \star \star}$ \\
\hline $\mathrm{Mg}$ & $0.08^{\star \star}$ & $0 \cdot 19^{\star \star \star}$ & $0 \cdot 15^{\star \star \star}$ & $0.23^{\star \star \star}$ & $-0.77^{\star \star \star}$ \\
\hline $\mathrm{Na}$ & $-0 \cdot 19^{\star \star \star}$ & $-0.19^{\star \star}$ & $-0 \cdot 10^{\star \star \star}$ & $0 \cdot 12^{\star \star \star}$ & $-0.52^{\star \star \star}$ \\
\hline K & $0 \cdot 12^{\star \star \star}$ & $0.26^{\star \star \star}$ & $0 \cdot 22^{\star \star \star}$ & $0.23^{\star * \star}$ & $-0 \cdot 81^{\star \star \star}$ \\
\hline$P$ & $-0 \cdot 12^{\star \star \star}$ & -0.02 & 0.01 & $0.09^{* *}$ & $-0.65^{\star \star \star}$ \\
\hline
\end{tabular}

$\mathrm{RCI}$, Recommendation Compliance Index; DQI-I, Diet Quality Index-International; DASH, Dietary Approaches to Stop Hypertension; MDS, Mediterranean Diet Score; DII, Dietary Inflammatory Index.

Values were significantly different: ${ }^{*} P<0.05,{ }^{* \star} P<0.01,{ }^{* \star *} P<0.001$.

$\dagger$ Difference in the number of cases is related to missing values for several variables. 
most of the vitamins and minerals, with the strongest correlations being observed between nutrient intakes and the DII.

\section{Associations between diet quality indices and risk biomarkers}

Table 3 summarises the independent associations between the selected diet quality indices and risk biomarkers.

Lipid biomarkers. The DQI-I and DASH scores exhibited a very similar pattern of significant inverse associations with lipid biomarkers. Both were inversely associated with total cholesterol, LDL-cholesterol, HDL-cholesterol, apo A1 and apo B (all $P<0.05)$. The RCI was inversely associated with total cholesterol, HDL-cholesterol and apo A1. The MDS was inversely associated with LDL-cholesterol and apo B.

Glycaemic biomarkers. Of the five selected diet quality indices, none exhibited an association with the glycaemic risk biomarkers after multivariate adjustment, with the exception of the RCI, which exhibited a positive association with insulin $(P<0 \cdot 05)$.

Metabolic markers. With increasing DQI-I and DASH scores, there was a significant linear decrease in central obesity as measured by waist circumference $(\beta=-0.009, P=0.036$ and $\beta=-0.011, P=0.005$, respectively). The DASH score and MDS were inversely associated with DBP $(\beta=-0.751$, $P=0.019$ and $\beta=-0.777$ and $P=0.014$, respectively). The MDS exhibited inverse associations with anaemic biomarkers. Participants with higher MDS had significantly lower levels of $\mathrm{Hb}$ and haematocrit.

Renal and hepatic function biomarkers. Concerning hepatic function as measured by liver enzymes (serum GOT, serum GPT and $\gamma$-GT), there were significant negative linear trends with the MDS, after additional statistical controlling for alcohol intake. Similarly, the Mediterranean dietary pattern was associated with significant linear decreases in creatinine and uric acid levels, irrespective of BMI. The RCI was inversely associated with creatinine levels.

\section{Stratification analyses}

Sex-specific stratification analyses showed that the observed associations of lipid biomarkers with the DQI-I and DASH scores were more significant in men than in women. The DII was inversely associated with plasma glucose, HbA1c, serum insulin and HOMA-IR in men aged $>50$ years. The DQI-I was also inversely associated with plasma glucose and HbA1c in only women (data not shown).

\section{Discussion}

Nutrition scientists have applied the diet quality concept to investigate the association between foods and nutrients and chronic disease incidence and mortality, based on prevailing hypotheses about the role of dietary factors in disease prevention $^{(36)}$. In the present nationwide cross-sectional study, the performance of five diet quality indices, based on their ability to detect significant independent variations in a range of chronic disease risk markers, was compared. Among the five selected diet quality indices, the MDS exhibited the strongest associations with numerous risk markers. Higher adherence to the Mediterranean dietary pattern was significantly associated with lower levels of LDL-cholesterol, apo B, DBP, traditional indicators of renal function (creatinine and uric acid) and liver enzymes (serum $\gamma$-GT and GPT), irrespective of age, sex, education level, smoking status, physical activity and daily energy intake.

Consistent with our findings, the health benefits of following a Mediterranean dietary pattern have been widely reported, in terms of increased survival ${ }^{(15)}$ and decreased risk of $\mathrm{CVD}^{(37)}$, diabetes $^{(38)}$, obesity and inflammation ${ }^{(39-41)}$. The advantage of the MDS is probably related to the expedient scoring method (i.e. points awarded to a sufficient number of beneficial components) and better fit to our data by capturing dietary variation related to cardiometabolic risk. In addition, the MDS is characterised by specific food and nutrient composition. High consumption of olive oil and nuts is a key contributor of the healthy aspects attributed to the Mediterranean diet ${ }^{(42)}$. This vegetable-based oil contains a high proportion of oleic acid (a MUFA, vitamin E, and antioxidant phenolic compounds ${ }^{(43)}$, which have been linked to a reduced risk of $\left.\mathrm{CVD}^{(44)}\right)$. Fish is an integral part of the Mediterranean diet, which has been reported to be inversely associated with CHD mortality ${ }^{(45)}$. In addition, the Mediterranean dietary pattern is characterised by moderate consumption of meat (particularly red processed meat), which may potentially affect plasma creatinine levels ${ }^{(46,47)}$. Serum creatinine and uric acid are simple measures and the most commonly used indicators of renal function in clinical practice. It must also be noted that the Mediterranean dietary pattern might be expected to fit another European population better than one in another part of the world where the ingredients would be scarce.

Both the DQI-I and DASH scores were inversely associated with LDL-cholesterol and total cholesterol and apo A1 and apo B. These associations with lipid biomarkers were compatible with the expectation, as dietary fat and SFA are common components in these diet quality scores. Kant \& Graubard ${ }^{(25)}$ have reported similar findings in comparing three dietary indices (Healthy Eating Index, Recommended Foods Score and Dietary Diversity Score for recommended foods) to predict cardiovascular risk. It is notable that the DQI-I and DASH scores were also inversely associated with HDL-cholesterol, which has also been observed in previous studies ${ }^{(16,48)}$. The probable explanation is that these scores were based on dietary recommendations targeting lower dietary fat intakes, hence reduced HDL-cholesterol levels in addition to total cholesterol and LDL-cholesterol levels, notably in those with higher baseline HDL-cholesterol levels ${ }^{(48)}$. The observation that reduced-fat diets lower HDL levels has led to controversy over the advisability of following lower-fat, higher-carbohydrate diets ${ }^{(49)}$.

Predictably, both the DQI-I and DASH diets were associated with a lower waist circumference, a relevant measure of abdominal obesity, and with decreased DBP. Our findings are consistent with previous literature documenting greater improvements in hypertension, cholesterol levels and weight loss in subjects following a DASH dietary pattern ${ }^{(16,50)}$.

No specific diet quality index was associated with glycaemic biomarkers in multivariable analyses controlling for age, sex 
Table 3. Multivariable linear regression of the associations between diet quality indices and risk markers, ORISCAV-LUX (Observation of Cardiovascular Risk Factors in Luxembourg) study, 2007-8† $(\beta$-Coefficients and $P$ values $)$

\begin{tabular}{|c|c|c|c|c|c|c|c|c|c|c|}
\hline & \multicolumn{2}{|c|}{$\mathrm{RCl}(n$ 1234) } & \multicolumn{2}{|c|}{ DQI-I (n 1206) } & \multicolumn{2}{|c|}{ DASH score $(n$ 1285) } & \multicolumn{2}{|c|}{ MDS ( $n$ 1352) } & \multicolumn{2}{|c|}{ DII ( $n$ 1352) } \\
\hline & $\beta$ & $P$ & $\beta$ & $P$ & $\beta$ & $P$ & $\beta$ & $P$ & $\beta$ & $P$ \\
\hline \multicolumn{11}{|l|}{ Lipid biomarkers ( $n$ 1040) } \\
\hline Total cholesterol $(\mathrm{mmol} / \mathrm{l})$ & -0.0697 & $0.045^{\star}$ & -0.0956 & 0.005 & $-0 \cdot 1225$ & $0.0001^{*}$ & -0.0508 & 0.10 & 0.0409 & 0.30 \\
\hline LDL-cholesterol (mmol/l) & -0.0003 & 0.25 & -0.0005 & $0.03^{*}$ & -0.0008 & $0.0006^{\star}$ & -0.0005 & $0.017^{\star}$ & 0.0003 & 0.33 \\
\hline HDL-cholesterol (mmol/li) & -0.0006 & $0.007^{*}$ & -0.0007 & $0.003^{*}$ & -0.0004 & $0.032^{*}$ & 0.0002 & 0.41 & 0 & 0.998 \\
\hline TAG (mmol/l) & 0.0002 & 0.25 & 0.0002 & 0.34 & 0.0001 & 0.45 & -0.00006 & 0.75 & -0.00003 & 0.89 \\
\hline Apo A1 (mg/l) & -0.21 & $0.0008^{*}$ & -0.19 & $0.002^{*}$ & -0.12 & $0.039^{*}$ & 0.01 & 0.84 & 0.02 & 0.75 \\
\hline Apo B (mg/l) & -0.09 & 0.30 & -0.19 & $0.03^{\star}$ & -0.26 & $0.002^{*}$ & -0.21 & $0.007^{*}$ & 0.13 & 0.21 \\
\hline \multicolumn{11}{|c|}{ Glycaemic biomarkers ( $n$ 1106) } \\
\hline Glucose $(\mathrm{mmol} / \mathrm{l})$ & -0.00001 & 0.96 & -0.0003 & 0.22 & -0.0002 & 0.22 & -0.00004 & 0.80 & -0.0002 & 0.32 \\
\hline $\mathrm{HbA1c}(\%)$ & -0.0007 & 0.71 & -0.002 & 0.34 & -0.002 & 0.30 & -0.0009 & 0.58 & -0.0001 & 0.96 \\
\hline HOMA-IR & 0.036 & 0.14 & -0.022 & 0.34 & -0.014 & 0.53 & -0.017 & 0.45 & -0.017 & 0.55 \\
\hline Insulin $(\mu \mathrm{g} / \mathrm{l})$ & 0.47 & $0.04^{*}$ & -0.13 & 0.57 & -0.06 & 0.80 & -0.10 & 0.65 & -0.22 & 0.41 \\
\hline \multicolumn{11}{|l|}{ Metabolic markers ( $n$ 1153) } \\
\hline $\mathrm{BMI}\left(\mathrm{kg} / \mathrm{m}^{2}\right)$ & 0.012 & $0.039^{*}$ & -0.004 & 0.43 & -0.007 & $0 \cdot 16$ & -0.002 & 0.68 & -0.003 & 0.67 \\
\hline WC (cm) & 0.005 & 0.23 & -0.009 & $0.04^{*}$ & -0.011 & $0.005^{\star}$ & -0.004 & 0.27 & 0.002 & 0.66 \\
\hline $\mathrm{SBP}(\mathrm{mmHg}) \ddagger$ & 0.0001 & 0.98 & 0.001 & 0.77 & -0.002 & 0.64 & -0.006 & 0.11 & -0.001 & 0.78 \\
\hline $\mathrm{DBP}(\mathrm{mmHg}) \ddagger$ & -0.36 & 0.32 & -0.289 & 0.40 & -0.751 & $0.02^{\star}$ & -0.777 & $0.01^{*}$ & 0.587 & 0.15 \\
\hline $\operatorname{CRP}(\mu \mathrm{g} / \mathrm{l})$ & -0.04 & 0.92 & 0.24 & 0.45 & 0.28 & 0.35 & 0.12 & 0.71 & 0.41 & 0.29 \\
\hline $\mathrm{Hb}(\mathrm{g} / \mathrm{l})$ & -0.26 & 0.45 & -0.29 & 0.38 & -0.30 & 0.35 & -0.92 & $0.003^{*}$ & 0.24 & 0.54 \\
\hline Haematocrit (\%) & -0.023 & 0.80 & -0.096 & 0.27 & -0.087 & 0.30 & -0.228 & $0.004^{*}$ & 0.054 & 0.60 \\
\hline \multicolumn{11}{|c|}{ Renal function biomarkers§ ( $n$ 1153) } \\
\hline Creatinine $(\mu \mathrm{mol} / \mathrm{l})$ & -0.796 & $0.03^{*}$ & -0.442 & 0.19 & -0.619 & 0.07 & -0.884 & $0.004^{*}$ & 0.442 & 0.27 \\
\hline Uric acid $(\mu \mathrm{mol} / \mathrm{l})$ & -0.595 & 0.78 & 0.952 & 0.64 & -1.309 & 0.52 & $-5 \cdot 175$ & $0.008^{*}$ & $-3 \cdot 271$ & 0.19 \\
\hline \multicolumn{11}{|c|}{ Hepatic function biomarkers $\|(n$ 1153) } \\
\hline$\gamma$-GT (mg/l) & -0.37 & 0.07 & -0.31 & 0.28 & -0.19 & 0.31 & -0.50 & $0.01^{*}$ & 0.84 & $0.0003^{*}$ \\
\hline GOT (mg/l) & 0.05 & 0.64 & 0.17 & 0.07 & 0.16 & 0.08 & -0.10 & 0.27 & -0.08 & 0.49 \\
\hline GPT (mg/l) & 0.01 & 0.93 & $0 \cdot 16$ & 0.26 & 0.03 & 0.83 & -0.31 & $0.02^{*}$ & $0 \cdot 10$ & 0.43 \\
\hline
\end{tabular}

RCI, Recommendation Compliance Index; DQI-I, Diet Quality Index-International; DASH, Dietary Approaches to Stop Hypertension; MDS, Mediterranean Diet Score; DII, Dietary Inflammatory Index; HbA1c, glycated Hb; HOMA-IR, index of insulin resistance and sensitivity calculated according to homeostasis model assessment; WC, waist circumference; SBP, systolic blood pressure; DBP, diastolic blood pressure; CRP, C-reactive protein; $\gamma$-GT, $\gamma$-glutamyl-transpeptidase; GOT, glutamate oxaloacetate transaminase; GPT, glutamate-pyruvate transaminase

† Non-fasting participants were excluded from the glycaemic and lipid biomarker models. Participants using antidiabetic, hypotensive and lipid-lowering medications were also excluded from the following outcome models, respectively: glucose; SBP and DBP; lipid biomarkers. Participants who were dieting for weigh loss were excluded from all he models. All ho models (RCl, DQl-I, DASH and MDS) were adjusted for age (continuous), sex, education level (primary, secondary or teriay), smokng sar

$\neq n 1007$ participants, after exclusion of those using hypotensive medications.

$\|$ The models were additionally controlled for alcohol intake (none, $<0-3$ and $<3$ drinks/d). 
and other potential confounders. The 20-year prospective Coronary Artery Risk Development in Young Adults (CARDIA) study also did not find any association between a diet consistent with the key American Dietary Guidelines and reduced risk of type 2 diabetes ${ }^{(51)}$, suggesting that most of the individual American Dietary Guideline recommendations have not been proven to reduce the risk of diabetes. Several diet quality scores, including MDS and DASH score, have been reported to be associated with a lower risk of type 2 diabetes in overweight subjects $^{(52)}$. In the present study population, ageand sex-specific stratification analyses showed that the DII was inversely associated with plasma glucose, HbA1c, serum insulin and HOMA-IR in men aged $>50$ years (data not shown). The DQI-I was also inversely associated with plasma glucose and HbA1c in only women. This sex-specific difference in cardiovascular risk prediction has been found in previous studies using different indices ${ }^{(26,53,54)}$. The absence of a significant association of diet quality indices with glycaemic biomarkers is probably explained by the relatively low prevalence of diabetes in the study population ${ }^{(30)}$; the small sample size did not allow to detect potentially significant changes in the risk of type 2 diabetes.

On the whole, the five diet quality indices were highly correlated in the study population, probably because most of these indices focus on dietary patterns rich in fruits and vegetables, whole grains, nuts and fish but poor in highsalt processed meats. The highest correlation was observed between the DASH and DQI-I scores, which confirms our findings with respect to the similarity in their ability to detect changes in lipid biomarkers. The inverse correlations between the DII and other diet quality indices are probably due to the fundamental difference in the conceptual background to construct this diet quality index, focusing on the inflammatory profile of the diet. Although these indices, in general, share the same beneficial and detrimental food or nutrient components, the observed variations in relation to biomarkers associated with disease risk are probably due to differences in their constructs and scoring criteria.

The RCI, DQI-I and DASH scores showed no direct correlations with important micronutrients such as vitamin D, Fe and I. This suggests that adherence to the respective dietary guidelines would be insufficient for preventing vitamin and mineral deficiencies, although other factors may influence the absorption; for example, vitamin D is not solely a foodbased nutrient. Furthermore, it is possible that the poor correlations arise from the inability of the FFQ to precisely assess the intake of these nutrients.

By contrast, the DII was strongly negatively correlated with virtually all the nutrients. Although the MDS was significantly correlated with daily Fe intake, finding low serum Fe and haematocrit levels among participants matching those observed on following a Mediterranean dietary pattern should be examined in other populations to determine whether the diet is really of poor Fe quality or whether further modifications and adaptations are required to be made in the MDS scoring system.

Surprisingly, the Luxembourg diet quality index measuring the compliance to the national dietary recommendations
(RCI) selected in the present study was associated with higher levels of serum insulin and BMI, but with lower levels of HDL and apo A1. The possibility of reverse causality, i.e. obese individuals and subjects with poor health status may have improved their dietary habits after being informed of a medical condition, is minimal, because these correlations were observed in a population sample relatively free from chronic diseases. Furthermore, participants taking medications or dieting for weight loss were excluded. In addition, further controlling for health awareness as expressed by the importance of eating a balanced diet did not change the results (data not shown). However, a bidirectional causal relationship between diet and health is possible, as diet quality has a positive effect on health and health influences diet quality and food choices. These unexpected findings indicate that adherence to the current national dietary guidelines may be insufficient to avoid cardiometabolic risk and disease.

Although the RCI helped to identify the major determinants of good compliance ${ }^{(10)}$, it is probably a poor indicator of risk prediction and not the ideal tool to reflect a good-quality healthy diet. The lack of consistent associations with risk biomarkers may be explained by the non-specificity of the type of protein and carbohydrate. The RCI emphasises a broad inclusion of many foods but not of micronutrients. Apart from population adherence to the national dietary guidelines, its ability to predict diet-related risk is questionable, and future modification and improvement of this indicator are required. In our national context, it is important to validate a summary nutritional indicator to serve as a useful tool for rapid screening, thereby aiding public health authorities to develop effective nutrition promotion messages to the general public ${ }^{(5,55)}$.

Similar to most of the nutrition population-based studies, potential limitations include factors related to the crosssectional design, which precludes inferences regarding causal relationships. In addition, an optimum dietary intake assessment strategy is still challenging in nutrition research ${ }^{(56)}$. The list of foods in a FFQ is a crucial element needed to capture the variability of dietary habits. The calculation of the selected diet quality indices relied on the 132-item FFQ. This tool has been shown to be sufficiently convenient and inexpensive to use in large-scale, population-based studies ${ }^{(57)}$, compared with a high-cost $24 \mathrm{~h}$ recall, which must be repeated to avoid under-reporting ${ }^{(58)}$, although both methods rely on cognitive processes such as memory and perception. It is worth mentioning that most of the selected indices were developed using the FFQ, except the DQI-I, which was originally designed using $24 \mathrm{~h}$ recalls. The short-term dietary intake assessment method was considered a weak point because food variety among the categories included in the DQI-I would have been prone to a misclassification error ${ }^{(17)}$. The ability of the DII to predict inflammation-related outcomes has been demonstrated using both recall methods and structured questionnaires ${ }^{(59)}$; however, the FFQ used in the present study seemed to be not ideal, as the unavailability of specific foods and nutrients probably contributed to the poor performance of the DII in the present study and, hence, hampered the detection of significant associations with risk biomarkers. 
The present study has several strong points. The data were derived from a recent nationwide sample of the general adult population. This is one of the few population-based studies that have directly compared alternative diet quality measures to determine whether one index can best predict the risk of chronic diseases. Previous attempts have been limited to comparison of different Mediterranean or American recommendation dietary scores ${ }^{(53)}$ with respect to specific health outcomes ${ }^{(26,52)}$. Applying these scores, developed in different settings, to a single European population is problematic, as it could be inadequate to assess the quality of the diet of one population based on the recommendations developed for another, especially when their eating habits differ substantially $^{(60)}$. The DII was empirically derived from a search of the literature that involved reading and scoring nearly 2000 articles that dealt with the relationship between dietary parameters and six inflammatory markers. The remaining three indices are based on dietary recommendations that, while generally sensible, are also culture bound. Nonetheless, the observed associations of each dietary pattern with various markers of chronic diseases provide evidence of their reproducibility and validity to a different extent.

In summary, the present study provides evidence that in the study population, the Mediterranean diet was associated with the most favourable biomarker risk profile, which supports the epidemiological application of the MDS in the future to evaluate diet-disease risk relationships. The Mediterranean diet serves potentially as a universal guideline for healthful food consumption, as it presents a healthy eating pattern associated with reduced levels of cardiometabolic, hepatic and renal biomarkers in an adult population. Considering the high multiple risk profile of the study population $^{(30)}$, our findings provide insights to determine whether the current dietary guidelines are truly helpful in the fight against chronic diseases.

\section{Supplementary material}

To view supplementary material for this article, please visit http://dx.doi.org/10.1017/S0007114514003456

\section{Acknowledgements}

A. A. was supported by a grant from the FNR (Fond National de Recherche) for the project (DIQUA-LUX, 5870404), Luxembourg. J. R. H. was supported by an Established Investigator Award in Cancer Prevention and Control from the Cancer Training Branch of the US National Cancer Institute (K05 CA136975). These funders had no role in the design and analysis of the study or in the writing of this article or in the interpretation of the results.

The authors' contributions are as follows: A. A. was involved in the conception and design of the ORISCAV-LUX survey, coordinated field data collection, conceived the present study, contributed to the statistical analyses and drafted the first version of the manuscript; C. V. performed the data analyses and contributed to the statistical analyses and data interpretation; G. E. C., N. Sauvageot and N. Shivappa contributed to the calculation of the diet quality indices and to the critical revision of the manuscript; J. R. H. provided expertise and oversight throughout the study. All authors approved the final version of the manuscript.

None of the authors has any conflicts of interest to declare.

\section{References}

1. Hu FB (2002) Dietary pattern analysis: a new direction in nutritional epidemiology. Curr Opin Lipidol 13, 3-9.

2. Wirt A \& Collins CE (2009) Diet quality - what is it and does it matter? Public Health Nutr 12, 2473-2492.

3. Arvaniti F \& Panagiotakos DB (2008) Healthy indexes in public health practice and research: a review. Crit Rev Food Sci Nutr 48, 317-327.

4. Patterson RE, Haines PS \& Popkin BM (1994) Diet quality index: capturing a multidimensional behavior. $J$ Am Diet Assoc 94, 57-64.

5. Kennedy ET, Ohls J, Carlson S, et al. (1995) The Healthy Eating Index: design and applications. J Am Diet Assoc 95, 1103-1108.

6. Haines PS, Siega-Riz AM \& Popkin BM (1999) The Diet Quality Index revised: a measurement instrument for populations. J Am Diet Assoc 99, 697-704.

7. Osler M, Heitmann BL, Gerdes LU, et al. (2001) Dietary patterns and mortality in Danish men and women: a prospective observational study. BrJ Nutr 85, 219-225.

8. Kant AK, Schatzkin A, Graubard BI, et al. (2000) A prospective study of diet quality and mortality in women. JAMA $\mathbf{2 8 3}$ 2109-2115.

9. Guenther PM, Reedy J \& Krebs-Smith SM (2008) Development of the Healthy Eating Index-2005. J Am Diet Assoc 108, 1896-1901.

10. Alkerwi A, Sauvageot N, Nau A, et al. (2012) Population compliance with national dietary recommendations and its determinants: findings from the ORISCAV-LUX study. $\mathrm{Br} J$ Nutr 108, 2083-2092.

11. Drescher LS, Thiele S \& Mensink GB (2007) A new index to measure healthy food diversity better reflects a healthy diet than traditional measures. J Nutr 137, 647-651.

12. Estaquio C, Kesse-Guyot E, Deschamps V, et al. (2009) Adherence to the French Programme National Nutrition Sante Guideline Score is associated with better nutrient intake and nutritional status. J Am Diet Assoc 109, 1031-1041.

13. Shatenstein B, Nadon S, Godin C, et al. (2005) Diet quality of Montreal-area adults needs improvement: estimates from a self-administered food frequency questionnaire furnishing a dietary indicator score. J Am Diet Assoc 105 1251-1260.

14. McNaughton SA, Ball K, Crawford D, et al. (2008) An index of diet and eating patterns is a valid measure of diet quality in an Australian population. J Nutr 138, 86-93.

15. Trichopoulou A, Costacou T, Bamia C, et al. (2003) Adherence to a Mediterranean diet and survival in a Greek population. N Engl J Med 348, 2599-2608.

16. Fung TT, Chiuve SE, McCullough ML, et al. (2008) Adherence to a DASH-style diet and risk of coronary heart disease and stroke in women. Arch Intern Med $\mathbf{1 6 8}$ 713-720.

17. Kim S, Haines PS, Siega-Riz AM, et al. (2003) The Diet Quality Index-International (DQI-I) provides an effective tool for cross-national comparison of diet quality as illustrated by China and the United States. J Nutr 133 3476-3484. 
18. Shivappa N, Steck SE, Hurley TG, et al. (2013) Designing and developing a literature-derived, population-based dietary inflammatory index. Public Health Nutr 17, 1689-1696.

19. Fransen HP \& Ocke MC (2008) Indices of diet quality. Curr Opin Clin Nutr Metab Care 11, 559-565.

20. Kant AK (1996) Indexes of overall diet quality: a review. J Am Diet Assoc 96, 785-791.

21. Kant AK (2004) Dietary patterns and health outcomes. J Am Diet Assoc 104, 615-635.

22. Alkerwi A (2014) Diet quality concept. Nutrition 30 , 613-618.

23. Coulston AM (2001) The search continues for a tool to evaluate dietary quality. Am J Clin Nutr $\mathbf{7 4}, 417$.

24. Hann CS, Rock CL, King I, et al. (2001) Validation of the Healthy Eating Index with use of plasma biomarkers in a clinical sample of women. Am J Clin Nutr 74, 479-486.

25. Kant AK \& Graubard BI (2005) A comparison of three dietary pattern indexes for predicting biomarkers of diet and disease. J Am Coll Nutr 24, 294-303.

26. Lassale C, Fezeu L, Andreeva VA, et al. (2012) Association between dietary scores and 13-year weight change and obesity risk in a French prospective cohort. Int J Obes (Lond) 36, 1455-1462.

27. Bach-Faig A, Geleva D, Carrasco J, et al. (2006) Evaluating associations between Mediterranean diet adherence indexes and biomarkers of diet and disease. Public Health Nutr 9, $1110-1117$.

28. Fung TT, Hu FB, Wu K, et al. (2010) The Mediterranean and Dietary Approaches to Stop Hypertension (DASH) diets and colorectal cancer. Am J Clin Nutr 92, 1429-1435.

29. Alkerwi A, Sauvageot N, Couffignal S, et al. (2010) Comparison of participants and non-participants to the ORISCAV-LUX population-based study on cardiovascular risk factors in Luxembourg. BMC Med Res Methodol 10, 80.

30. Alkerwi A, Sauvageot N, Donneau AF, et al. (2010) First nationwide survey on cardiovascular risk factors in GrandDuchy of Luxembourg (ORISCAV-LUX). BMC Public Health 10, 468.

31. Sauvageot N, Alkerwi A, Albert A, et al. (2013) Use of food frequency questionnaire to assess relationships between dietary habits and cardiovascular risk factors in NESCAV study: validation with biomarkers. Nutr J 12, 143.

32. Sauvageot N, Alkerwi A, Adelin A, et al. (2013) Validation of the food frequency questionnaire used to assess the association between dietary habits and cardiovascular risk factors in the NESCAV study. J Nutr Food Sci 3, 208.

33. Hercberg S (2005) Tables de composition des aliments SU.VI.MAX (French food composition databank from the SU.VI.MAX study). Paris: Economica.

34. Alkerwi A, Donneau AF, Sauvageot N, et al. (2011) Dietary, behavioural and socio-economic determinants of the metabolic syndrome among adults in Luxembourg: findings from the ORISCAV-LUX study. Public Health Nutr $\mathbf{1 5}$ 849-859.

35. Hagstromer M, Oja P \& Sjostrom M (2006) The International Physical Activity Questionnaire (IPAQ): a study of concurrent and construct validity. Public Health Nutr 9, $755-762$.

36. Kant AK (2010) Dietary patterns: biomarkers and chronic disease risk. Appl Physiol Nutr Metab 35, 199-206.

37. Martinez-Gonzalez MA \& Sanchez-Villegas A (2004) The emerging role of Mediterranean diets in cardiovascular epidemiology: monounsaturated fats, olive oil, red wine or the whole pattern? Eur J Epidemiol 19, 9-13.

38. Martinez-Gonzalez MA, de la Fuente-Arrillaga C, NunezCordoba JM, et al. (2008) Adherence to Mediterranean diet and risk of developing diabetes: prospective cohort study. BMJ 336, 1348-1351.

39. Fung TT, McCullough ML, Newby PK, et al. (2005) Dietquality scores and plasma concentrations of markers of inflammation and endothelial dysfunction. Am J Clin Nutr 82, 163-173.

40. Fragopoulou E, Panagiotakos DB, Pitsavos C, et al. (2010) The association between adherence to the Mediterranean diet and adiponectin levels among healthy adults: the ATTICA study. J Nutr Biochem 21, 285-289.

41. Sofi F, Cesari F, Abbate R, et al. (2008) Adherence to Mediterranean diet and health status: meta-analysis. BMJ 337, a1344.

42. Tur JA, Romaguera D \& Pons A (2005) The Diet Quality IndexInternational (DQI-I): is it a useful tool to evaluate the quality of the Mediterranean diet? Br J Nutr 93, 369-376.

43. Owen RW, Giacosa A, Hull WE, et al. (2000) Olive-oil consumption and health: the possible role of antioxidants. Lancet Oncol 1, 107-112.

44. Manson JE, Gaziano JM, Jonas MA, et al. (1993) Antioxidants and cardiovascular disease: a review. J Am Coll Nutr 12, 426-432.

45. Hu FB, Bronner L, Willett WC, et al. (2002) Fish and omega-3 fatty acid intake and risk of coronary heart disease in women. JAMA 287, 1815-1821.

46. Jacobsen FK, Christensen CK, Mogensen CE, et al. (1979) Pronounced increase in serum creatinine concentration after eating cooked meat. Br Med J 1, 1049-1050.

47. Nair S, O'Brien SV, Hayden K, et al. (2014) Effect of a cooked meat meal on serum creatinine and estimated glomerular filtration rate in diabetes-related kidney disease. Diabetes Care 37, 483-487.

48. Obarzanek E, Sacks FM, Vollmer WM, et al. (2001) Effects on blood lipids of a blood pressure-lowering diet: the Dietary Approaches to Stop Hypertension (DASH) trial. Am J Clin Nutr 74, 80-89.

49. Katan MB, Grundy SM \& Willett WC (1997) Should a low-fat, high-carbohydrate diet be recommended for everyone? Beyond low-fat diets. $N$ Engl J Med 337, 563-566, discussion $566-567$.

50. Fitzgerald KC, Chiuve SE, Buring JE, et al. (2012) Comparison of associations of adherence to a Dietary Approaches to Stop Hypertension (DASH)-style diet with risks of cardiovascular disease and venous thromboembolism. $J$ Thromb Haemost 10, 189-198.

51. Zamora D, Gordon-Larsen P, He K, et al. (2011) Are the 2005 Dietary Guidelines for Americans associated with reduced risk of type 2 diabetes and cardiometabolic risk factors? Twenty-year findings from the CARDIA study. Diabetes Care 34, 1183-1185.

52. de Koning L, Chiuve SE, Fung TT, et al. (2011) Diet-quality scores and the risk of type 2 diabetes in men. Diabetes Care 34, 1150-1156.

53. McCullough ML, Feskanich D, Stampfer MJ, et al. (2002) Diet quality and major chronic disease risk in men and women: moving toward improved dietary guidance. Am J Clin Nutr 76, 1261-1271.

54. Nicklas TA, O'Neil CE \& Fulgoni VL 3rd (2012) Diet quality is inversely related to cardiovascular risk factors in adults. J Nutr 142, 2112-2118.

55. McCabe-Sellers BJ, Bowman S, Stuff JE, et al. (2007) Assessment of the diet quality of US adults in the Lower Mississippi Delta. Am J Clin Nutr 86, 697-706.

56. Kristal AR, Peters U \& Potter JD (2005) Is it time to abandon the food frequency questionnaire? Cancer Epidemiol Biomarkers Prev 14, 2826-2828. 
57. Schatzkin A, Kipnis V, Carroll RJ, et al. (2003) A comparison of a food frequency questionnaire with a 24-hour recall for use in an epidemiological cohort study: results from the biomarker-based Observing Protein and Energy Nutrition (OPEN) study. Int J Epidemiol 32, 1054-1062.

58. Ma Y, Olendzki BC, Pagoto SL, et al. (2009) Number of 24-hour diet recalls needed to estimate energy intake. Ann Epidemiol 19, 553-559.
59. Shivappa N, Steck SE, Hurley TG, et al. (2013) A populationbased dietary inflammatory index predicts levels of C-reactive protein in the Seasonal Variation of Blood Cholesterol Study (SEASONS). Public Health Nutr 17, 1825-1833.

60. Dubois L, Girard M \& Bergeron N (2000) The choice of a diet quality indicator to evaluate the nutritional health of populations. Public Health Nutr 3, 357-365. 